\title{
A Critical Review on Empirical Head-Predicting Models of Two-phase Petroleum Fluids in Electrical Submersible Pumps
}

\author{
Mohammadzaheri M*and Ghodsi M \\ Mechanical and Industrial Engineering Department, Sultan Qaboos University, Oman \\ *Corresponding author: Morteza Mohammadzaheri, Mechanical and Industrial \\ Engineering Department, Sultan Qaboos University, AlKhoud, Oman, Tel: 98942834; \\ Email:morteza@alumni.adelaide.edu.au
}

\section{Mini Review \\ Volume 2 Issue 4}

Received Date: April 17, 2018

Published Date: May 09, 2018

\section{Abstract}

This paper critically reviews empirical models, which predict head of two-phase petroleum fluids in electrical submersible pumps. The article categorises empirical models in terms of mathematical structure and parameter identification algorithm. Categories are heuristic and artificial intelligence models. Models of the former category have fairly low accuracy and 4 or fewer parameters identifiable using non-iterative methods; conversely, models the of latter category have high accuracy and tens or even hundreds of parameters. These models require complex iterative identification algorithms. Due to availability of inexpensive digital processors, use of accurate artificial intelligence models is anticipated to broaden.

Keywords: Electrical Submersible Pump; Petroleum; Multi-layer Perceptron; Head; Fuzzy

\section{Introduction}

Electrical Submersible Pumps (ESP) are commonly used in downhole operations for a wide range of flow rates [1]. These pumps are known as effective and economical devices to lift large volume of fluid under different well conditions [2]. However, use of over- or under-size ESPs lead to premature equipment failure or low petroleum fluid recovery, respectively [3]. As a result, size selection of ESPs is a crucial task. In the case that liquid is pumped, suitability of ESP size is assessed using manufacturer curves, which depict head versus flow rate. Such curves, however, are inapplicable, when an ESP pumps two-phase petroleum fluid with high gas content. The alternative is to develop models predicting head of two-phase fluids in ESPs based on related variables. These models are called 'head predicting' models and have been investigated since 1980's [4]. In addition to headpredicting, some other models have also been developed for side purposes e.g. to determine flow pattern, gas bubble size or in-situ gas volume fraction in ESPs; such models are outside the scope of this paper [5-7].

Three main approaches have been employed to develop head-predicting models for two-phase petroleum fluids in ESPs: Analytical, Numerical and Empirical. The 


\section{Petroleum \& Petrochemical Engineering Journal}

basis of analytical (also known as mechanistic) and numerical approaches is to solve conservation equations. Solution of these equations demand availability of closure relationships to estimate bubble size, drag force coefficient, in-situ gas void fractions, friction factors etc [8]. Moreover, analytical models largely trust unrealistic assumptions and/or oversimplification of complex physics of two-phase flows within the ESP [4,5,9-11]. Numerical models also have their own peculiar weak points; they are formulated based on one-dimensional two-fluid conservations of mass and momentum along streamlines and require the prediction of surging initiation [12]. Therefore, analytical and numerical models (except for oversimplified homogenous model [3]) are yet to be practically used, and empirical models are widely trusted alternatively [13,14].This paper particularly addresses empirical models.

\section{Overview of Empirical Models}

Empirical models can be categorised in two groups: (i) Heuristic models and (ii) Artificial Intelligence (AI) models. Each has specifications in terms of mathematical structure and parameter identification. In this paper, models developed for two-phase petroleum fluids or similar mixtures are discussed only, and models developed for water-gas fluids are excluded. Moreover, it is assumed that models have been developed for a nominal rotational speed, and affinity laws are used to extend the use of model to other rotational speeds, unless otherwise stated [13].

All empirical models have three input variables from the following table, where the output is the estimated head of two-phase fluid, $\hat{H}_{m}$.

\begin{tabular}{|c|c|c|c|}
\hline Symbol & Variable & Symbol & Variable \\
\hline$p_{i n}$ & input pressure & $\alpha$ & gas void fraction \\
\hline$\rho_{\lambda}$ & liquid density & $q_{l}$ & liquid volumetric flow rate \\
\hline$\rho_{\gamma}$ & gas density & $q_{g,}$ & gas volumetric flow rate \\
\hline$\rho_{\mu}$ & mixture density & $q_{m}$ & mixture volumetric flow rate \\
\hline
\end{tabular}

Table 1: Most common input variables to a head-predicting model of two-phase petroleum fluids in ESPs.

Only input variable outside Table 1 is rotational speed, $\Omega$, which has been used as the fourth input of a model [14]. Maximum head and flow rate of the pump, Hmax and qmax, have been used as parameters (not variables) in a model [13]. Another parameter is $\mathrm{Hl}$, the head estimated by manufacturer curve, if the whole fluid was liquid.

\section{Heuristic Empirical models}

The first model, Model 1, was developed by Turpin et al in 1986 [15]:

$$
\hat{H}_{m}=H_{l} \exp \left(346430\left(\frac{q_{g}}{p_{\text {in }} q_{l}}\right)^{2}-410\left(\frac{q_{g}}{p_{\text {in }} q_{l}}\right)\right),
$$

Then, Model 2 was proposed by Sachdeva et al, in 1992 [16]:

$$
\hat{H}_{m}=\frac{K_{2}}{\rho_{m} g} p_{i n}^{{ }^{E}} \alpha^{E 2} q_{l}^{E 3} .
$$

Model 3 was presented by Zhou and Sachdeva in 2010 [13]:

$$
\hat{H}_{m}=H_{\max } K_{3}\left(C p_{\text {in }}\right)^{\alpha E_{4}}(1-\alpha) E_{5}\left(1-\frac{q_{m}}{q_{\max }}\right)^{E_{6}},
$$

where $C$ is pressure unit coefficient, e.g. 1,1000 or 0.145 for psi,ksi or $\mathrm{kPa}$. The values of E1, E2,E3, E4, E5, E6, K2 and $\mathrm{K} 3$ are listed in Zhou D, et al. [13] for multiple stages of electrical submersible pumps.

Model 3 seems to be a modified version of Model 2. In this model, when gas void fraction and flow rate equal zero, estimated head is Hmax. The values of E1, E2, E3 and $\mathrm{K} 2$ are listed in for multiple stages of electrical submersible pumps [13].

Models (1-3) are converted to linear equations after taking logarithm. As a result, the parameters can be identified with use of experimental data and non-iterative least square of error algorithm [17] (or alternatively with response surface method (RSM) [18] or full factorial experiments $[19,20])$. The number of identifiable 


\section{Petroleum \& Petrochemical Engineering Journal}

parameters in Models 1, 2 and 3 are 2, 4 and 4 respectively.

\section{Artificial Intelligence Models}

Four AI models have been reported so far to predict head of two-phase fluids in ESPs. In all of them, parameters were identified using experimental data and iterative algorithms. The first model was a Multi-Layer Perceptron (MLP), reported in 2015 [4]:

$$
\hat{H}_{m}=\sum_{j=1}^{7} \mathbf{T}_{j}\left[2\left(1+\exp \left(-2\left(\mathbf{W}_{1 j} p_{i n}+\mathbf{W}_{2 j} q_{m}+\mathbf{W}_{3 j} \alpha+\mathbf{b}_{j}\right)\right)\right)^{-1}-1\right]+c .
$$

Matrices $\mathbf{W}_{3 \times 7}, \mathbf{T}_{1 \times 7}$ and $\mathbf{b}$ and scalar $c$ are parameters of Model 4, equivalent to 36 scalar parameters in total. It was shown that (4) outperforms Models (1-3) by far in terms of estimation accuracy, as detailed in Table 2 [4].

Following this work, a more complex version of was presented with an extra input variable of rotational speed, $\Omega$. In this model, a history of input variables were used instead of individual variables [4,14]. Later on, a Radial Basis Function Network (RBFN)with 390 parameters and a fuzzy models with 30 parameters were also successfully developed to predict head of two-phase fluids in ESPs $[3,18]$. It was also shown that RBFN and fuzzy models present much smaller test estimation errors than all heuristic models (Table 2).

\section{Summary and Conclusion}

Empirical head-predicting models of two-phase petroleum fluids in ESPs were reviewed and categorised as heuristic and artificial intelligence (AI) models. Table 2 presents number of parameters and the absolute test error of the models estimating the head of 8 stages of I42B radial ESP. The tests error is an average of all test errors reported in for the same ESP. Heuristic ones have the advantage of small number of parameters and noniterative parameter identification method $[1,3,4,18]$. However, AI models present much smaller error at the cost of higher number of parameters and complex iterative parameter identification algorithms. With availability of inexpensive digital processors, AI models are expected to find wider use in the future.

\begin{tabular}{|c|c|c|c|c|c|c|}
\hline & \multicolumn{3}{|c|}{ Heuristic Models } & \multicolumn{3}{c|}{ AI Models } \\
\hline Model & $\mathbf{1}$ & $\mathbf{2}$ & $\mathbf{3}$ & ANN[4] & Fuzzy[18] & RBFN[3] \\
\hline Number of Parameters & 2 & 4 & 4 & 36 & 30 & 390 \\
\hline
\end{tabular}

Table 2: Number of parameters and mean of absolute test error in different empirical models of two-phase petroleum fluids in ESPs.

\section{References}

1. Mohammadzaheri M, Tafreshi R, Khan Z, Franchek M, Grigoriadis K (2016) An intelligent approach to optimize multiphase subsea oil fields lifted by electrical submersible pumps. Journal of Computational Science 15: 50-59.

2. $\quad$ Bai Y, Bai Q (2012) Subsea engineering handbook. $1^{\text {st }}$ (Edn.), Gulf Professional Publishing, pp: 956.

3. Mohammadzaheri M, Ghodsi M, AlQallaf A (2018) Estimate of the head produced by electrical submersible pumps on gaseous petroleum fluids, a raidal basis function netrwork approach. International Journal of Artificial Intelligence and Applications 9: 53-62.
4. Mohammadzaheri M, Tafreshi R, Khan Z, Franchek M, Grigoriadis K (2015) Modelling of Petroleum Multiphase fluids in ESPs, an Intelliegnt Approach. Offshore Mediternean Conference, Ravenna, Italy.

5. Barrios L, Prado MG (2011) Modeling Two-Phase Flow Inside an Electrical Submersible Pump Stage. Journal of Energy Resources Technology 133(4): pp 042902.

6. Zhu J, Zhang HQ (2017) Numerical Study on Electrical-Submersible-Pump Two-Phase Performance and Bubble-Size Modeling. SPE Production \& Operations 32(3).

7. Zhu J, Zhang HQ (2016) Mechanistic modeling and numerical simulation of in-situ gas void fraction inside ESP impeller. Journal of Natural Gas Science and Engineering 36: 144-154. 


\section{Petroleum \& Petrochemical Engineering Journal}

8. Zhu J, Zhang HQ (2018) A Review of Experiments and Modeling of Gas-Liquid Flow in Electrical Submersible Pumps. Energies 11(1): 180.

9. Sun D, Prado M (2005) Modeling gas-liquid head performance of electrical submersible pumps. Journal of Pressure Vessel Technology 127(1): 31-38.

10. Sun D (2003) Modeling Gas-Liquid Head Performance of Electrical Submersible Pumps. The University of Tulsa 64(3): 219 .

11. Sachdeva RD, Schmidt Z (1988) Two-Phase Flow ThroughElectrical Submersible Pumps. dissertation The University of Tulsa, Oklahoma.

12. Zhu J, Guo X, Liang F, Zhang HQ (2017) Experimental study and mechanistic modeling of pressure surging in electrical submersible pump. Journal of Natural Gas Science and Engineering 45: 625-636.

13. Zhou D, Sachdeva R (2010) Simple model of electric submersible pump in gassy well. Journal of Petroleum Science and Engineering 70(3-4): 204-213.

14. Pineda LR, Serpa AL, Biazussi JL, Sassim NA (2016) Operational Control of an Electrical Submersible Pump Working with Gas-Liquid Flow Using Artificial Neural Network. presented at the IASTED International Conference on Intelligent Systems and Control Campinas, Brazil.

15. Turpin JL, Lea JF, Bearden JL (1986) Gas-Liquid Flow Through Centrifugal Pumps-Correlation of Data," presented at the The Third International Pump Symposium, Texas, USA pp: 13-20.

16. Sachdeva R, Doty D, Schmidt Z (1992) Performance of Axial Electric Submersible Pumps in a Gassy Well. SPE Rocky Mountain Regional Meeting.

17. Mohammadzaheri M, Chen L, Ghaffari A, Willison J (2009) A combination of linear and nonlinear activation functions in neural networks for modeling a de-superheater. Simulation Modelling Practice and Theory 17(2): 398-407.

18. Ghodsi M (2015) Optimization of mover acceleration in DC tubular linear direct-drive machine using response surface method. International Review of Electrical Engineering 10: 492-500.

19. Ghodsi M, Mirzamohamadi S, Talebian S, Hojjat Y, Sheikhi M, et al. (2015) Analytical, numerical and experimental investigation of a giant magnetostrictive (GM) force sensor. Sensor Review 35(4): 357-365.

20. Ghodsi M, Hosseinzadeh N, Ozer A, Dizaj HR, Hojjat Y, et al. (2017) Development of Gasoline Direct Injector using giant magnetostrictive materials. IEEE Transactions on Industry Applications 53(1): 521529.

21. Qallaf A, Mohammadzaheri M (2017) A Fuzzy Model to Estimate Head of Gaseous Petroleum Fluids Driven by Electrical Submersible Pumps. The Engineering and Technology, Computer, Basic and Applied Sciences, Sydney, Australia. 